\title{
RESTRICTED UNIFORM BOUNDEDNESS IN BANACH SPACES
}

\author{
OLAV NYGAARD AND MÄRT PÕLDVERE
}

\begin{abstract}
Precise conditions for a subset $A$ of a Banach space $X$ are known in order that pointwise bounded on $A$ sequences of bounded linear functionals on $X$ are uniformly bounded. In this paper, we study such conditions under the extra assumption that the functionals belong to a given linear subspace $\Gamma$ of $X^{*}$. When $\Gamma=X^{*}$, these conditions are known to be the same ones assuring a bounded linear operator into $X$, having $A$ in its image, to be onto. We prove that, for $A$, deciding uniform boundedness of sequences in $\Gamma$ is the same property as deciding surjectivity for certain classes of operators.
\end{abstract}

\section{INTRODUCTION}

Let $X$ be a real or complex Banach space, let $\Gamma$ be a linear subspace of the dual space $X^{*}$, and let $A$ be a subset of $X$. We shall say that the set $A$ is $\Gamma$-boundedness deciding if every pointwise bounded on $A$ family $\mathcal{F} \subset \Gamma$ is pointwise bounded on the whole of $X$ (and thus norm bounded). Note that, in this definition, we may assume the families $\mathcal{F}$ to be sequences.

Two natural situations are when $\Gamma=X^{*}$ or when the space $X$ is a dual, say $X=Z^{*}$ for some Banach space $Z$ and $\Gamma=Z$, in which cases boundednessdecidingness is well understood. The key concepts in these cases, respectively, are the notions of thickness and weak*-thickness (which resemble the second Baire category) introduced by Kadets and Fonf $[\mathrm{KF}]$. Recall that a set $B \subset X$ (respectively, $B \subset X=Z^{*}$ ) is said to be norming (respectively, weak*-norming) if

$$
\left.\inf _{x^{*} \in S_{X^{*}}} \sup _{x \in B}\left|x^{*}(x)\right|>0 \quad \text { (respectively, } \inf _{z \in S_{Z}} \sup _{z^{*} \in B}\left|z^{*}(z)\right|>0\right),
$$

or, equivalently, if the closed (respectively, weak*-closed) absolutely convex hull of $B$ contains a ball; otherwise $B$ is said to be non-norming (respectively, weak ${ }^{*}$ non-norming). The set $A$ is said to be thick (respectively, weak ${ }^{*}$-thick) if it can not be represented as a non-decreasing union of non-norming (respectively, weak*non-norming) sets; otherwise $A$ is said to be thin (respectively, weak ${ }^{*}$-thin).

We refer to the survey article [N2] for detailed sources of the following two omnibus-theorems.

Theorem 1.1. Let $A$ be a subset of a Banach space $X$. The following assertions are equivalent.

(a) The set $A$ is thick.

(b) Whenever a sequence of functionals in the dual space $X^{*}$ is pointwise bounded on $A$, then this sequence is norm bounded (i.e., $A$ is $X^{*}$-boundedness deciding).

2000 Mathematics Subject Classification. 46B20, 46A30.

Key words and phrases. Uniform boundedness, thick set, boundedness deciding set.

The second named author was supported by Estonian Science Foundation Grant 7308. 
(b) Whenever a family of continuous linear operators from the space $X$ to some Banach space is pointwise bounded on $A$, then this family is norm bounded.

(c) Whenever $Y$ is a Banach space and $T: Y \rightarrow X$ is a continuous linear operator such that $T[Y] \supset A$, then $T[Y]=X$.

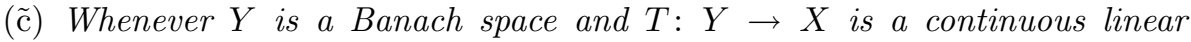
injection with $T\left[B_{Y}\right]$ being a closed set such that $T[Y] \supset A$, then $T[Y]=X$.

(d) Whenever $(\Omega, \Sigma, \mu)$ is a measure space and an essentially separably valued function $g: \Omega \rightarrow X^{*}$ is such that $a \circ g \in L_{1}(\mu)$ for every $a \in A$, then $x \circ g \in L_{1}(\mu)$ for every $x \in X$.

(e) The linear span of $A$ is dense and barrelled.

Theorem 1.2. Let $Z$ be a Banach space and let $A$ be a subset of the dual space $Z^{*}$. The following assertions are equivalent.

(a) The set $A$ is weak*-thick.

(b) Whenever a sequence of elements of the space $Z$ is pointwise bounded on $A$, then this sequence is norm bounded (i.e., $A$ is $Z$-boundedness deciding).

(六) Whenever a family of dual continuous linear operators from $Z^{*}$ to some dual Banach space $Y^{*}$ is pointwise bounded on $A$, then this family is norm bounded.

(c) Whenever $Y$ is a Banach space and $T: Z \rightarrow Y$ is a continuous linear operator such that $T^{*}\left[Y^{*}\right] \supset A$, then $T^{*}\left[Y^{*}\right]=Z^{*}$.

(d) Whenever $(\Omega, \Sigma, \mu)$ is a measure space and an essentially separably valued function $g: \Omega \rightarrow Z$ is such that $a \circ g \in L_{1}(\mu)$ for every $a \in A$, then $z^{*} \circ g \in L_{1}(\mu)$ for every $z^{*} \in Z^{*}$.

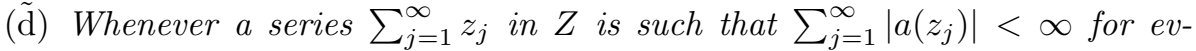
ery $a \in A$, then this series is weakly unconditionally Cauchy, that is, $\sum_{j=1}^{\infty}\left|z^{*}\left(z_{j}\right)\right|<\infty$ for every $z^{*} \in Z^{*}$.

The objective of this paper is to create a concept $-\Gamma$-thickness - which contains both thickness and weak*-thickness, and to show how the equivalences (a)-(d) of both Theorems 1.1 and 1.2 can be formulated in a unified setting.

In Section 2, we generalize the equivalences of Theorems 1.1 and 1.2 involving thickness and boundedness to Theorem 2.6 giving the equivalence of $\Gamma$-thickness and $\Gamma$-boundedness decidingness. It follows that if $\Gamma_{1}$ and $\Gamma_{2}$ are linear subspaces of $X^{*}$, then $\Gamma_{1}$-thickness and $\Gamma_{2}$-thickness are the same if and only if the norm closures of $\Gamma_{1}$ and $\Gamma_{2}$ coincide. It also follows that $\Gamma$-thickness it just weak ${ }^{*}$-thickness in another setting (Corollary 2.9) and thus the equivalence of $\Gamma$-thickness and certain integrability decidingness readily follows from Theorem 1.2.

In Section 3, our focus will be on formulating theorems that contain as particular cases the equivalences between thickness and surjectivity in Theorems 1.1 and 1.2.

Our notation is mostly standard. The closed unit ball and the unit sphere of a Banach space $X$, and the natural embedding into the bidual $X^{* *}$ are denoted, respectively, by $B_{X}$ and $S_{X}$, and $j_{X}$. For a set $A \subset X$, we denote by $\operatorname{span}(A)$ the linear span of $A$, and by $\operatorname{absconv}(A)$ its absolutely convex hull. If $Y$ is a Banach space (over the same scalar field as $X)$, then $\mathcal{L}(Y, X)$ will stand for the Banach space of continuous linear operators from $Y$ to $X$. If some subsets $A_{n} \subset X, n \in \mathbb{N}$, are such that $A_{1} \subset A_{2} \subset A_{3} \subset \ldots$, then, for their union, we sometimes write $\bigcup_{n=1}^{\infty} A_{n} \uparrow$. 


\section{THICK AND THIN SETS, BOUNDEDNESS, AND INTEGRABILITY}

Throughout the section, $X$ will be a Banach space and $\Gamma$ a linear subspace of $X^{*}$. We shall write $\tau$ for the weak topology $\sigma(X, \Gamma)$ on $X$. In this situation, one has $(X, \tau)^{*}=\Gamma$ (see, e.g, [M, page 207, Theorem 2.4.11]).

Let us first define the main concepts we shall need. We shall say that a subset $B \subset X$ is $\Gamma$-norming if

$$
\inf \left\{\sup _{x \in B}\left|x^{*}(x)\right|: x^{*} \in S_{X^{*}} \cap \Gamma\right\}>0 .
$$

The set $B$ will be said to be $\Gamma$-non-norming if it is not $\Gamma$-norming. We shall say that a set $A \subset X$ is $\Gamma$-thick if it can not be represented as a non-decreasing countable union of $\Gamma$-non-norming sets, i.e., whenever $A=\bigcup_{n=1}^{\infty} A_{n} \uparrow$, then, for some $m \in \mathbb{N}$, the set $A_{m}$ is $\Gamma$-norming. The set $A$ will be said to be $\Gamma$-thin if it is not $\Gamma$-thick.

Remark 2.1. Note that a subset of $X$ is $\Gamma$-norming if and only if its absolutely convex hull is $\Gamma$-norming.

Remark 2.2. Note that a bounded subset of $X$ is $\Gamma$-norming if and only if it is $\bar{\Gamma}$-norming (norm-closure in $X^{*}$ ).

Remark 2.3. Suppose that $A$ is $\Gamma$-thin. Then it can be represented as $A=$ $\bigcup_{n=1}^{\infty} A_{n} \uparrow$, where the $A_{n}, n \in \mathbb{N}$, are $\Gamma$-non-norming sets. One also has $A=$ $\bigcup_{n=1}^{\infty} A_{n} \cap n B_{X} \uparrow$. Thus, a $\Gamma$-thin set can be represented as a countable nondecreasing union of norm-bounded $\Gamma$-non-norming sets.

The following lemma is a simple application of the Hahn-Banach separation theorem in the locally convex space $(X, \tau)$. It will be used extensively throughout the paper.

Lemma 2.4. A subset $B$ of $X$ is $\Gamma$-norming if and only if there exists some $\delta>0$ such that $\overline{a b \operatorname{conv}}^{\tau}(B) \supset \delta B_{X}$.

Proof. If $\overline{\operatorname{absconv}}^{\tau}(B) \supset \delta B_{X}$ and $x^{*} \in S_{X^{*}} \cap \Gamma$, then

$$
\sup _{x \in B}\left|x^{*}(x)\right|=\sup \left\{\left|x^{*}(x)\right|: x \in \overline{\operatorname{absconv}}^{\tau}(B)\right\} \geq \sup _{x \in \delta B_{X}}\left|x^{*}(x)\right|=\delta .
$$

Conversely, if, for every $n \in \mathbb{N}$, one has $\overline{\operatorname{absconv}}^{\tau}(B) \not \supset \frac{1}{n} B_{X}$, then there is a sequence $\left(x_{n}\right)_{n=1}^{\infty} \subset X$ such that $\left\|x_{n}\right\| \leq \frac{1}{n}$ and $x_{n} \notin \overline{\operatorname{absconv}}^{\tau}(B)$ for all $n \in \mathbb{N}$. By the Hahn-Banach separation theorem (see, e.g, [M, page 180, Theorem 2.2.28] for its possibly non-Hausdorff version), for each $n \in \mathbb{N}$, there is an $x_{n}^{*} \in(X, \tau)^{*}=\Gamma$ with $\left\|x_{n}^{*}\right\|=1$ such that

$$
\begin{aligned}
\operatorname{Re} x_{n}^{*}\left(x_{n}\right) & >\sup \left\{\operatorname{Re} x_{n}^{*}(x): x \in \overline{\operatorname{absconv}}^{\tau}(B)\right\} \\
& =\sup \left\{\left|x_{n}^{*}(x)\right|: x \in \overline{\operatorname{absconv}}^{\tau}(B)\right\}=\sup _{x \in B}\left|x_{n}^{*}(x)\right|
\end{aligned}
$$

and thus $\frac{1}{n}>\sup _{x \in B}\left|x_{n}^{*}(x)\right|$. The latter clearly implies that $\inf \left\{\sup _{x \in B}\left|x^{*}(x)\right|: x^{*} \in\right.$ $\left.S_{X^{*}} \cap \Gamma\right\}=0$.

A better result than Remark 2.1 follows.

Corollary 2.5. A subset $B$ of $X$ is $\Gamma$-norming if and only if $\overline{a b s c o n v}^{\tau}(B)$ is $\Gamma$ norming. 
We now arrive at a generalization of the equivalences $(\mathrm{a}) \Leftrightarrow(\mathrm{b})$ of Theorems 1.1 and 1.2 .

Theorem 2.6. Let $A \subset X$. The following assertions are equivalent.

(a) The set $A$ is $\Gamma$-thick.

(b) The set $A$ is $\Gamma$-boundedness deciding.

Proof. $(\mathrm{a}) \Rightarrow(\mathrm{b})$. Suppose that $A$ is $\Gamma$-thick and let a family $\mathcal{F} \subset \Gamma$ be pointwise bounded on $A$, i.e., $\sup _{x^{*} \in \mathcal{F}}\left|x^{*}(a)\right|<\infty$ for all $a \in A$. Putting $A_{n}=\{a \in A$ : $\left.\sup _{x^{*} \in \mathcal{F}}\left|x^{*}(a)\right| \leq n\right\}, n \in \mathbb{N}$, one has $A=\bigcup_{n=1}^{\infty} A_{n} \uparrow$. Since $A$ is $\Gamma$-thick, there are $m \in \mathbb{N}$ and $\delta>0$ such that $\overline{\operatorname{absconv}}^{\tau}\left(A_{m}\right) \supset \delta B_{X}$, thus $\sup _{x^{*} \in \mathcal{F}}\left|x^{*}(x)\right| \leq \frac{m}{\delta}\|x\|$ for all $x \in X$.

(b) $\Rightarrow(\mathrm{a})$. Suppose that $A$ is $\Gamma$-thin. Then we can write $A=\bigcup_{n=1}^{\infty} A_{n} \uparrow$ where, for each $n \in \mathbb{N}$, the set $A_{n}$ is $\Gamma$-non-norming and thus we can find $x_{n}^{*} \in S_{X^{*}} \cap \Gamma$ with $\sup _{x \in A_{n}}\left|x_{n}^{*}(x)\right|<1 / n$. The unbounded sequence $\left(n x_{n}^{*}\right)$ is pointwise bounded on $A$.

Corollary 2.7. Let $x^{*} \in X^{*} \backslash \bar{\Gamma}$. Then $\operatorname{ker} x^{*}$ is $\Gamma$-thick.

Proof. Suppose that a family $\mathcal{F} \subset \Gamma$ is pointwise bounded on $\operatorname{ker} x^{*}$. Since $\left(\operatorname{span}\left\{x^{*}\right\}\right)^{\perp}$ $=\left(\operatorname{ker} x^{*}\right)^{\perp \perp}$ is canonically isometrically isomorphic to $\left(\operatorname{ker} x^{*}\right)^{* *}$ and, by the Banach-Steinhaus uniform boundedness principle, every Banach space $Z$ is a $Z^{*}$ boundedness deciding subset of its bidual, $\mathcal{F}$ is pointwise bounded also on $\left(\operatorname{span}\left\{x^{*}\right\}\right)^{\perp} \subset$ $X^{* *}$. Since, for the closed subspace $V:=\bar{\Gamma} \oplus \operatorname{span}\left\{x^{*}\right\}$ of $X^{*}$, one has $V^{*}=$ $(\bar{\Gamma})^{\perp} \oplus\left(\operatorname{span}\left\{x^{*}\right\}\right)^{\perp}$, it follows that $\mathcal{F}$ is pointwise bounded on $V^{*}$, and, again by the uniform boundedness principle, norm bounded. Thus, by Theorem 2.6, $\operatorname{ker} x^{*}$ is $\Gamma$-thick.

Theorem 2.8. Let $\Gamma_{1}$ and $\Gamma_{2}$ be linear subspaces of $X^{*}$. Then $\Gamma_{1}$-thick sets and $\Gamma_{2}$-thick sets are the same if and only if the norm closures of $\Gamma_{1}$ and $\Gamma_{2}$ coincide.

Proof. Necessity is clear from Corollary 2.7. Sufficiency follows from Remarks 2.3 and 2.2 .

As a particular case: Thickness and weak*-thickness are the same only for reflexive spaces.

Comparing the equivalences $(\mathrm{a}) \Leftrightarrow(\mathrm{b})$ of Theorems 1.2 and 2.6 immediately gives

Corollary 2.9. Let $A \subset X$ and let $i: \bar{\Gamma} \rightarrow X^{*}$ be the natural embedding. The following assertions are equivalent.

(a) The set $A$ is $\Gamma$-thick.

(b) The set $i^{*} j_{X}[A]$ is weak $k^{*}$-thick (as a subset of $\bar{\Gamma}^{*}=\Gamma^{*}$ ).

The following result contains the equivalences $(\mathrm{a}) \Leftrightarrow(\mathrm{d})$ and $(\mathrm{a}) \Leftrightarrow(\mathrm{d}) \Leftrightarrow(\tilde{\mathrm{d}})$ of Theorems 1.1 and 1.2, respectively.

Theorem 2.10. Let $A \subset X$. The following assertions are equivalent to each other and to (a) of Theorem 2.6.

(d) Whenever $(\Omega, \Sigma, \mu)$ is a measure space and $g: \Omega \rightarrow \bar{\Gamma}$ is an essentially separable valued function such that $a \circ g \in L_{1}(\mu)$ for every $a \in A$, then $x^{* *} \circ g \in L_{1}(\mu)$ for every $x^{* *} \in X^{* *}$. 
(d') Whenever $(\Omega, \Sigma, \mu)$ is a measure space and $g: \Omega \rightarrow \bar{\Gamma}$ is an essentially separable valued function such that $a \circ g \in L_{1}(\mu)$ for every $a \in A$, then $x \circ g \in L_{1}(\mu)$ for every $x \in X$.

(更) Whenever a sequence $\left(x_{j}^{*}\right)_{j=1}^{\infty} \subset \bar{\Gamma}$ is such that $\sum_{j=1}^{\infty}\left|x_{j}^{*}(a)\right|<\infty$ for every $a \in A$, then $\sum_{j=1}^{\infty}\left|x^{* *}\left(x_{j}^{*}\right)\right|<\infty$ for every $x^{* *} \in X^{* *}$.

( $\left.\tilde{\mathrm{d}}^{\prime}\right)$ Whenever a sequence $\left(x_{j}^{*}\right)_{j=1}^{\infty} \subset \bar{\Gamma}$ is such that $\sum_{j=1}^{\infty}\left|x_{j}^{*}(a)\right|<\infty$ for every $a \in A$, then $\sum_{j=1}^{\infty}\left|x_{j}^{*}(x)\right|<\infty$ for every $x \in X$.

Proof. $(\mathrm{d}) \Rightarrow\left(\mathrm{d}^{\prime}\right) \Rightarrow\left(\tilde{\mathrm{d}}^{\prime}\right)$ and $(\mathrm{d}) \Rightarrow(\tilde{\mathrm{d}}) \Rightarrow\left(\tilde{\mathrm{d}}^{\prime}\right)$ are obvious. $(\mathrm{a}) \Rightarrow(\mathrm{d})$ and $(\tilde{\mathrm{d}}) \Rightarrow($ a) follow from the corresponding implications of Theorem 1.2 via Corollary 2.9. Since, by the Banach-Steinhaus uniform boundedness principle, $j_{X}[X]$ is a weak ${ }^{*}$-thick subset of $X^{* *},\left(\tilde{\mathrm{d}}^{\prime}\right) \Rightarrow(\tilde{\mathrm{d}})$ follows from the implication $(\mathrm{a}) \Rightarrow(\tilde{\mathrm{d}})$ of Theorem 1.2 .

Remark 2.11. In the assertion ( $(\tilde{\mathrm{d}})$ (and thus also in (d)) of Theorem 1.2, $Z$ can be replaced by its norm dense subspace (this is clear from Remarks 2.3 and 2.2, and [ANP, Lemma 2.1 and its proof]). Thus $\bar{\Gamma}$ can be replaced by $\Gamma$ in Theorem 2.10.

3. More on the Relationship Between $\Gamma$-NORMing And $\Gamma$-Thick SETS, AND A CHARACTERIZATION OF THICK SETS BY SURJECTIVITY OF OPERATORS

Throughout this section, $X$ will be a Banach space, $\Gamma \subset X^{*}$ will be a linear subspace, $\tau$ will be the weak topology $\sigma(X, \Gamma)$ on $X$, and $A \subset X$.

By definition, $\Gamma$-thick sets form a subclass among the $\Gamma$-norming sets. Theorems 2.6 and 2.10 characterize the subclass of $\Gamma$-thick sets by the properties of determining boundedness and integrability. In this section, $\Gamma$-thick sets will be characterized as sets determining surjectivity of certain classes of operators, loosely spoken. To this end, let us first make some general observations about $\Gamma$-thick and $\Gamma$-thin sets.

As we already remarked, the set $A$ is $\Gamma$-norming if and only if its absolutely convex hull is. The same is true for $\Gamma$-thick sets.

Proposition 3.1. The following assertions are equivalent.

(a) The set $A$ is $\Gamma$-thick.

(b) $\operatorname{absconv}(A)$ is $\Gamma$-thick.

(c) $\operatorname{span}(A)$ is $\Gamma$-thick.

Proof. The implications (a) $\Rightarrow$ (b) and (b) $\Rightarrow$ (c) are obvious. The implication (c) $\Rightarrow$ (a) follows from Theorem 2.6 because a family $\mathcal{F} \subset \Gamma$ is pointwise bounded on $A$ if and only if it is pointwise bounded on $\operatorname{span}(A)$.

By adapting a construction from $[\mathrm{KF}]$ and $[\mathrm{N} 1]$, we next prove a rather general result about how $\Gamma$-normingness and $\Gamma$-thickness are related.

Theorem 3.2. The following assertions are equivalent.

(a) The set $A$ is $\Gamma$-thick.

(b) Every set $B \subset X$ with $A \subset \operatorname{span}(B)$ is $\Gamma$-norming.

More precisely, if $A$ is $\Gamma$-thin, then there exists an absolutely convex normbounded but $\Gamma$-non-norming set $B$ such that $A \subset \operatorname{span}(B)$. If $S_{X^{*}} \cap \Gamma$ is weak*norming, then this absolutely convex norm-bounded but $\Gamma$-non-norming set $B$ can be chosen to be $\tau$-closed. 
Proof. The implication (a) $\Rightarrow(\mathrm{b})$ follows from Proposition 3.1 , (a) $\Leftrightarrow(\mathrm{c})$, since every $\Gamma$-thick set is $\Gamma$-norming. For $(\mathrm{b}) \Rightarrow(\mathrm{a})$, suppose that $A$ is $\Gamma$-thin. Then it has a representation $A=\bigcup_{n=1}^{\infty} A_{n} \uparrow$ where $\left(A_{n}\right)_{n=1}^{\infty}$ is a non-decreasing sequence of $\Gamma$-non-norming sets with $A_{n} \subset n B_{X}, n \in \mathbb{N}$ (see Remark 2.3). Put

$$
C_{1}=A_{1} \quad \text { and } \quad C_{n}=\frac{1}{n^{2}}\left(A_{n} \backslash A_{n-1}\right), \quad n \in \mathbb{N}, n \geq 2 .
$$

Letting $B=\bigcup_{n=1}^{\infty} C_{n}$, one clearly has $A \subset \operatorname{span}(B)$, and it remains to show that $B$ is $\Gamma$-non-norming. To this end, let $\varepsilon>0$ be arbitrary. We are going to find an $x^{*} \in S_{X^{*}} \cap \Gamma$ such that $\sup _{x \in B}\left|x^{*}(x)\right| \leq \varepsilon$. To this end, choose $m \in \mathbb{N}$ such that $\frac{1}{m}<\varepsilon$. Since the set $A_{m}$ is $\Gamma$-non-norming, there is an $x^{*} \in S_{X^{*}} \cap \Gamma$ such that $\sup _{x \in A_{m}}\left|x^{*}(x)\right|<\varepsilon$. Now fix an arbitrary $u \in B$ and choose $k \in \mathbb{N}$ such that $u \in C_{k}$. There are two possibilities: either $k \leq m$ or $k>m$. In the first case

$$
\left|x^{*}(u)\right| \leq \sup _{x \in C_{k}}\left|x^{*}(x)\right| \leq \sup _{x \in A_{k}}\left|x^{*}(x)\right| \leq \sup _{x \in A_{m}}\left|x^{*}(x)\right|<\varepsilon .
$$

In the second case, when $k>m$, one has, since $C_{k} \subset \frac{1}{k} B_{X}$,

$$
\left|x^{*}(u)\right| \leq \sup _{x \in C_{k}}\left|x^{*}(x)\right| \leq \frac{1}{k}<\frac{1}{m}<\varepsilon .
$$

It follows that $\sup _{x \in B}\left|x^{*}(x)\right| \leq \varepsilon$, and, since $\varepsilon$ was arbitrary, $B$ is $\Gamma$-non-norming.

For the "more precisely" part, just notice that, for the set $B$ above, the bounded set $\operatorname{absconv}(B)$ is $\Gamma$-non-norming. By Corollary 2.5, also $\overline{\operatorname{absconv}}^{\tau}(B)$ is $\Gamma$-nonnorming, and, if $S_{X^{*}} \cap \Gamma$ is weak*-norming, it is still bounded. Indeed, assume that $S_{X^{*}} \cap \Gamma$ is weak*-norming, and suppose for contradiction that the $\tau$-closure $\bar{C}^{\tau}$ of a (norm) bounded set $C \subset X$ is not bounded. By Lemma $2.4, \overline{\text { absconv }} w^{*}\left(S_{X^{*}} \cap\right.$ $\Gamma) \supset \delta B_{X^{*}}$ for some $\delta>0$. Letting $N \in \mathbb{N}$ be arbitrary, there is some $z \in \bar{C}^{\tau}$ with $\|z\|>\frac{N}{\delta}$. Choosing a $z^{*} \in S_{X^{*}} \cap \Gamma$ such that $\left|z^{*}\left(\frac{z}{\|z\|}\right)\right|>\frac{\delta}{2}$, one has $\left|z^{*}(z)\right|>\frac{\delta}{2}\|z\|>\frac{N}{2}$, and thus, picking $x \in C$ with $\left|z^{*}(z)-z^{*}(x)\right|<\frac{N}{4}$,

$$
\|x\| \geq\left|z^{*}(x)\right| \geq\left|z^{*}(z)\right|-\left|z^{*}(z)-z^{*}(x)\right|>\frac{N}{2}-\frac{N}{4}=\frac{N}{4} .
$$

Since $N$ was arbitrary, this contradicts the boundedness of $C$.

We next translate Theorem 3.2 into a general theorem about surjectivity of bounded operators. For this, when $Y$ is a Banach space over the same scalar field as $X$, let $\mathcal{L}^{\tau}(Y, X)$ denote the set of operators $T$ in $\mathcal{L}(Y, X)$ with the property that

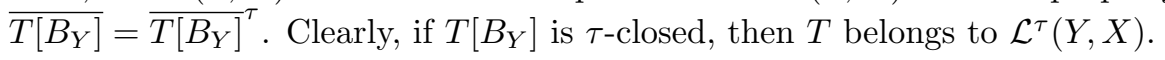

Theorem 3.3. The following assertions are related as $(\mathrm{a}) \Rightarrow(\mathrm{c}) \Rightarrow(\tilde{\mathrm{c}})$.

(a) The set $A$ is $\Gamma$-thick.

(c) Whenever $Y$ is a Banach space, every $T \in \mathcal{L}^{\tau}(Y, X)$ with $T[Y] \supset A$ is onto.

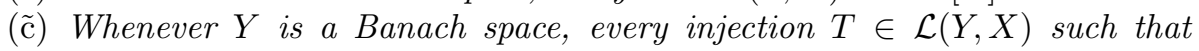
$T\left[B_{Y}\right]$ is $\tau$-closed with $T[Y] \supset A$ is onto.

If $S_{X^{*}} \cap \Gamma$ is weak ${ }^{*}$-norming, then these assertions are equivalent.

Proof. $(\mathrm{a}) \Rightarrow(\mathrm{c})$. Assume that $A$ is $\Gamma$-thick. Let $Y$ be a Banach space and let $T \in \mathcal{L}^{\tau}(Y, X)$ be such that $T[Y] \supset A$. By a classical lemma of Banach (see, e.g., [HHZ, page 67, Lemma 82]), it suffices to show that $\overline{T\left[B_{Y}\right]} \supset \gamma B_{X}$ for some $\gamma>0$. Since $T[Y] \supset A$, also $T[Y]$ is $\Gamma$-thick, and thus, since $T[Y]=\bigcup_{n=1}^{\infty} n T\left[B_{Y}\right]$, there 
is some $m \in \mathbb{N}$ for which the set $m T\left[B_{Y}\right]$ is $\Gamma$-norming, i.e., there is a $\delta>0$ such that $m \overline{T\left[B_{Y}\right]^{\tau}} \supset \delta B_{X}$. Since $T \in \mathcal{L}^{\tau}(Y, X)$, we obtain $\overline{T\left[B_{Y}\right]} \supset \frac{\delta}{m} B_{X}$, as desired.

(c) $\Rightarrow(\tilde{\mathrm{c}})$ is obvious since if $T\left[B_{Y}\right]$ is $\tau$-closed, then $T$ belongs to $\mathcal{L}^{\tau}(Y, X)$.

Now assume that $S_{X^{*}} \cap \Gamma$ is weak $^{*}$-norming.

$(\tilde{\mathrm{c}}) \Rightarrow(\mathrm{a})$. Suppose that $A$ is $\Gamma$-thin. By Theorem 3.2, there is a $\tau$-closed absolutely convex norm-bounded but $\Gamma$-non-norming set $B \subset X$ such that $\operatorname{span}(B) \supset A$. Clearly $B$ is also norm-closed. Letting $Y$ be the linear span of $B$ normed by the Minkowski functional of $B$, i.e., $\|y\|=\inf \left\{\gamma>0: \frac{1}{\gamma} y \in B\right\}, y \in Y$, it is well-known that $Y$ is a Banach space with $B_{Y}=B$ (see, e.g., [M, pages 330-331, the proof of Lemma 3.4.28]). Define $T: Y \rightarrow X$ to be the natural embedding. Then $T\left[B_{Y}\right]=B$ is $\tau$-closed and $T[Y]=\operatorname{span} B \supset A$. On the other hand, since $B$ is $\Gamma$-thin, $\operatorname{span} B$ is $\Gamma$-thin as well, and thus $T[Y]=\operatorname{span} B \varsubsetneqq X$, i.e., $T$ is not onto.

Remark 3.4. Theorem 3.3 contains Theorem 1.1, (a) $\Leftrightarrow(\mathrm{c}) \Leftrightarrow(\tilde{\mathrm{c}})$, since if $\Gamma=X^{*}$, then $\tau$ is the weak topology $w$, and, by Mazur's theorem, $\mathcal{L}^{w}(Y, X)=\mathcal{L}(Y, X)$.

In the setting of Theorem 1.2, one has to consider, in Theorem 3.3, $X=Z^{*}$, the dual subspace $\Gamma$ is $Z$ (considered as a subspace of $Z^{* *}=X^{*}$ ), the topology $\tau$ is the weak* topology $w^{*}$ on $Z^{*}$, and $\mathcal{L}^{\tau}(Y, X)=\mathcal{L}^{w^{*}}\left(Y, Z^{*}\right)$ is the set of the operators $T \in \mathcal{L}\left(Y, Z^{*}\right)$ for which $\overline{T\left[B_{Y}\right]} w^{*}=\overline{T\left[B_{Y}\right]}$. Whenever $T \in \mathcal{L}(Z, Y)$, the adjoint operator $T^{*}$ is in $\mathcal{L}^{w^{*}}\left(Y^{*}, Z^{*}\right)$ (indeed, $T^{*}$ is weak ${ }^{*}$-to-weak ${ }^{*}$ continuous, thus $T^{*}\left[B_{Y^{*}}\right]$ is weak* compact (because $B_{Y^{*}}$ is weak* compact), hence weak* closed, and consequently $\overline{T^{*}\left[B_{Y^{*}} w^{*}\right.}=\overline{T^{*}\left[B_{Y^{*}}\right]}$ ). The implication (a) $\Rightarrow$ (c) of Theorem 1.2 now follows from Theorem $3.3,(\mathrm{a}) \Rightarrow(\mathrm{c})$.

For the implication (c) $\Rightarrow(\mathrm{a})$ of Theorem 1.2, suppose that the set $A \subset Z^{*}$ is not weak*-thick. One has to show that there is a Banach space $Y$ and an operator in $\mathcal{L}(Z, Y)$ whose adjoint fails to be surjective but its range contains $A$.

If $M:=\overline{\operatorname{span}}^{w^{*}}(A) \neq Z^{*}$, then, letting $q: Z \rightarrow Z / M_{\perp}=: Y$ be the quotient mapping, one has $q^{*}\left[Y^{*}\right]=M$.

Now suppose that $\operatorname{\operatorname {span}}^{w^{*}}(A)=Z^{*}$. Theorem 3.2 produces an absolutely convex norm-bounded but $Z$-non-norming set $B \subset Z^{*}$ such that $A \subset \operatorname{span}(B)$. Then $p(z)=\sup _{z^{*} \in B}\left|z^{*}(z)\right|, z \in Z$, is a norm on $Z$ (because $\operatorname{span}(B)$ is weak*-dense). Letting $Y$ be a completion of $Z$ with respect to $p$, and $J:(Z,\|\cdot\|) \rightarrow Y$ the natural inclusion, one has $J \in \mathcal{L}((Z,\|\cdot\|), Y)$ (because $B$ is norm-bounded), with $J^{*}$ being non-surjective (because the range of $J$ is not closed - otherwise, by the Banach inverse mapping theorem, the formal identity $i:(Z, p) \rightarrow(Z,\|\cdot\|)$ would be continuous which is not the case due to the weak ${ }^{*}$-non-normingness of $B$ and thus the range of $J^{*}$ is not closed) but $J^{*}\left[Y^{*}\right] \supset A$. Indeed, each $a \in B$ is in $(Z, p)^{*}$. Fixing an arbitrary $a \in A$, one also has $a \in(Z, p)^{*}$ (because $A \subset \operatorname{span}(B)$ ), and thus $a$ extends uniquely to some $y^{*} \in Y^{*}$. But now, for every $z \in Z$, one has $J^{*} y^{*}(z)=y^{*}(J z)=a(z)$ meaning that $J^{*} y^{*}=a$.

The implication (c) $\Rightarrow$ (a) of Theorem 1.2 (as one could just observe) is not a direct consequence of Theorem 3.3. The following general result on $\Gamma$-thickness containing the equivalences $(\mathrm{a}) \Leftrightarrow(\mathrm{c})$ of both Theorems 1.2 and 1.1 is an immediate consequence of Corollary 2.9 and this equivalence of Theorem 1.2 itself. As before, we assume that $\Gamma$ is a linear subspace of $X^{*}$ and $A \subset X$.

Theorem 3.5. Let $i: \bar{\Gamma} \rightarrow X^{*}$ be the natural embedding. The following assertions are equivalent. 
(a) The set $A$ is $\Gamma$-thick.

(c) Whenever $Y$ is a Banach space, for every $T \in \mathcal{L}(\bar{\Gamma}, Y)$ with $T^{*}\left[Y^{*}\right] \supset$ $i^{*} j_{X}[A]$, the adjoint $T^{*}$ is onto.

In the setting of Theorem 1.2, $X=Z^{*}, \Gamma=j_{Z}[Z] \subset Z^{* *}=X^{*}$, and $j_{X}=j_{Z^{*}}$; thus Theorem 1.2, (a) $\Leftrightarrow(\mathrm{c})$, is a particular case of Theorem 3.5.

For Theorem 1.1, (a) $\Rightarrow(\mathrm{c})$, suppose that $A \subset X$ is thick ( $=X^{*}$-thick), and let a Banach space $Y$ and $T \in \mathcal{L}(Y, X)$ be such that $T[Y] \supset A$. Then also $T^{* *}\left[Y^{* *}\right] \supset$ $j_{X}[A]$, and, by Theorem 3.5 (or, since $j_{X}[A]$ is a weak ${ }^{*}$-thick subset of $X^{* *}$, just by Theorem $1.2,(\mathrm{a}) \Leftrightarrow(\mathrm{c})), T^{* *}$ is onto, and thus also $T$ is onto.

For (c) $\Rightarrow(\mathrm{a})$, suppose that $A \subset X$ is such that, whenever $Y$ is a Banach space, every member of $\mathcal{L}(Y, X)$ whose range contains $A$ is onto, and let a Banach space $Y$ and $T \in \mathcal{L}\left(X^{*}, Y\right)$ satisfy $T^{*}\left[Y^{*}\right] \supset j_{X}[A]$. By Theorem 3.5, it suffices to show that, in fact, $T^{*}\left[Y^{*}\right]=X^{* *}$. To this end, observe that $V:=\left\{y^{*} \in Y^{*}: T^{*} y^{*} \in j_{X}[X]\right\}$ is a closed subspace of $Y^{*}$. Letting the operator $S \in \mathcal{L}(V, X)$ be defined by $j_{X} S y^{*}=$ $T^{*} y^{*}, y^{*} \in V$, one has $S[V] \supset A$, and, by our assumption, in fact, $S[V] \supset X$; thus $T^{*}\left[Y^{*}\right] \supset j_{X}[X]$. The Banach-Steinhaus uniform boundedness principle tells us that $j_{X}[X]$ is a weak*-thick subset of $X^{* *}$, and it follows that $T^{*}\left[Y^{*}\right]=X^{* *}$, as desired.

\section{REFERENCES}

[ANP] T. A. Abrahamsen, O. NygaArd, and M. Põldvere, On weak integrability and boundedness in Banach spaces, J. Math. Anal. Appl. 314 (2006), 67-74.

[HHZ] P. Habala, P. HÁJeK, ANd V. Zizler, Introduction to Banach spaces, I, Charles University, Prague, 1996.

[KF] M. I. KADETS AND V. P. Fonf, Two theorems on the massiveness of the boundary in a reflexive Banach space (in Russian), Funktsional. Anal. i Prilozhen. 17 (1983), 77-78; English translation in: Funct. Anal. Appl. 17 (1983), 227-228.

[M] R. E. Megginson. An Introduction to Banach Space Theory, Graduate Texts in Mathematics, 183, Springer, New York, 1998.

[N1] O. NygaARD, Boundedness and surjectivity in normed spaces, Int. J. Math. Math. Sci. 32 (2002), 149-165.

[N2] O. NyGAARD, Thick sets in Banach spaces and their properties, Quaest. Math. 29 (2006), $59-72$.

Department of Mathematics, University of Agder, Servicebox 422, 4604 Kristiansand, NORWAY

E-mail address: Olav.Nygaard@uia.no

$U R L:$ http://home.hia.no/ olavn/

Institute of Mathematics, University of Tartu, J. Liivi 2, 50409 Tartu, Estonia

E-mail address: mart.poldvere@ut.ee 